\title{
Identidad y juventud en la cibercultura desde una perspectiva educativa $^{1}$
}

\author{
Ana Elena Schalk Quintanar ${ }^{2}$
}

\begin{abstract}
En este artículo se presenta una propuesta que integra tres variables: el proceso de identidad de los jóvenes, la cibercultura en la cual se desarrollan y los desafíos que estas variables plantean a la educación. Primeramente, se requiere comprender el concepto de "juventud" desde su espacio históricocultural y no como un concepto generalizado. Junto con ello, abordar su proceso de construcción de la identidad, en una búsqueda equilibrada de la relación del "yo y los otros" en el entorno de la cibercultura, que hace difusos los límites entre "lo real" y "lo imaginario" y que, a su vez, constituye el espacio de interacción, de validación individual y de difusión de conocimiento. Además, se reflexiona cómo la cibercultura, a través de algunos de los fenómenos que se abordan en este artículo, impacta de forma directa en el proceso de identidad de los jóvenes. Responder a la pregunta respecto de cuáles son los espacios de acción y de posibilidad de los educadores y de los sistemas educativos en el fortalecimiento de las bases que permitan una construcción de identidad que, por un lado, potencie los elementos de valor de la cultura actual y, por otro, fortalezca aquello que los jóvenes necesitan para desenvolverse en ella, constituye el propósito central de este artículo.
\end{abstract}

Palabras Clave: Jóvenes, cibercultura, identidad, educación.

\section{Identity and Youth in Cyberculture from education perspective}

\section{ABSTRACT}

This article presents a proposal integrating three variables: identity process of young people, cyberculture in which they are developed and challenges that these variables impose to education. First, it is necessary to understand the concept of youth from its historical-cultural space and not as a generalized concept. Along with this, it is also a need to address the process

1 Artículo recibido el 15/09/2018. Artículo aprobado el 30/11/2018

2 Mexicana, Académica e investigadora. Advisory Board Member at The Education Partners. Docente del Curso de Directivos de la Organización de Estados Iberoamericanos (OEA). E-mail:maschalk@gmail.com 
of identity construction, in a balanced searching for the relationship between "me and others," in the environment of cyberculture, which diffuses boundaries between what is "real" and what is "imaginary," and that, in turn, constitutes a space for interaction, individual validation and dissemination of knowledge. In addition, this study is aimed at reflecting through some phenomena addressed in this article, on how cyberculture directly impacts on the identity process of young people. This article is focused in answering the question about what are the areas of action and possibilities for educators and education systems in strengthening the bases that allow an identity construction for enhancing the value elements of today's culture on the one hand, and strengthen what young people need to develop, on the other.

Keywords: Youth, Ciberculture, Identity and Education

\section{Identidade e Juventude na Cibercultura desde uma perspectiva educativa}

\section{RESUMO}

Neste artigo apresenta-se uma proposta que integra três variáveis: o processo de identidade dos jovens, a cibercultura na qual se desenvolvem, e os desafios que estas variáveis apresentam à educação. Primeiramente, precisa-se compreender o conceito de juventude desde seu espaço histórico-cultural e não como um conceito generalizado. Junto com aquilo, abordar seu processo de construção da identidade, em uma busca equilibrada da relação do "eu e os outros", no entorno da cibercultura, que faz difusos os limites entre "o real" e "o imaginário", e que ao mesmo tempo constitui o espaço de interação, validação individual e de difusão de conhecimento. Além disso, reflete-se sobre como a cibercultura, através de alguns dos fenômenos que se abordam neste artigo, impacta de forma direta no processo de identidade dos jovens. Responder à pergunta a respeito de quais são os espaços de ação e de possibilidade dos educadores e dos sistemas educativos no fortalecimento das bases que permitem uma construção de identidade que, por um lado, potencie os elementos de valor da cultura atual e, por outro, fortaleça aquilo que os jovens necessitam para agirem nela, constitui o propósito central deste artigo.

Palavras-chave: Jovens, Cibercultura, Identidade, Educação

\section{Construcción de identidad de los nativos digitales}

En los primeros años de este nuevo siglo se acuñó, como resultado de un poco más de dos décadas de reflexiones al respecto, la definición explícita de los "nativos digitales" (Prensky, 2001) y su 
conceptualización incluía una contrastación genérica de éstos respecto de las generaciones anteriores. Su distinción principal es su alta comprensión del lenguaje digital y utilizar diferentes tipos de dispositivos (en ocasiones al mismo tiempo) para acceder tanto a la información como al entretenimiento. Tapscott los denomina la "Net Generation", y Howe y Strauss como los “Millenials", quienes además se destacan por su necesidad de compartir información, tener un ritmo de vida más rápido y ser consumidores de contenidos (Georges \& Veytia, 2017).

Sin embargo, a pesar de compartir algunas condiciones generales, existen elementos muy distintos que caracterizan a estos nativos digitales (Georges \& Veytia, 2017), por lo que es importante señalar que es propósito de este artículo hacer planteamientos más cercanos a la realidad de los jóvenes en Latinoamérica, más que una reflexión generalizada de la interacción de los tres elementos propuestos: identidad, cibercultura y educación. Es importante tener en cuenta que lo juvenil, en tanto cultura, no representa una categoría unívoca pues está culturalmente construida y por tanto está necesariamente vinculada a los contextos sociohistóricos, producto de las relaciones de fuerza de una determinada sociedad (Abril \& León, 2016).

El concepto de juventud debe ser pensado en el marco del complejo entramado de relaciones sociales e históricas atravesadas por el género, la clase social, la cultura, la localización geográfica. Por lo tanto, superando las cuestiones biológicas y cronológicas, que nunca son homogéneas ni universales (Chaves, 2010, Reguillo, 2000), se propone pensar a las jóvenes "como seres en relación" (Lemus, 2017).

En contraposición a estos nativos, se encuentran las generaciones anteriores, (los migrantes), quienes se han enfrentado al desafío de integrar la tecnología a sus formas y estilos de vida. Solo algunos pocos profesores en las salas de clase hoy en día son nativos digitales y, seguramente, con el paso de los años, esto se incrementará significativamente, es decir, nativos digitales enseñarán 
a generaciones que seguramente se irán transformando pero que comparten la base de lo "digital" como parte de sus vidas 3 . Sin embargo, en los últimos años, este fenómeno de migrantes digitales enseñando a nativos ha invertido, por primera vez en la historia de la educación, la relación educador-educado, lo cual, en el contexto cultural de América Latina, ha tenido un desarrollo que merece atención.

Esto ha producido un sinfín de fenómenos, algunos de los cuales no hemos sido siquiera capaces de identificar; en otras ocasiones los hemos visto pero no hemos podido anticiparnos, y, en otras, lo hemos hecho, pero el ritmo de cambio no ha ido a la par de las necesidades del contexto. Sin dejar de valorar los esfuerzos y las experiencias acertadas que existen en las iniciativas individuales y colectivas, el punto de atención que permite la definición de desafíos y la conducción de decisiones oportunas en la educación es precisamente la capacidad de mirar y de valorar críticamente aquello que está pendiente por hacer.

En ese sentido, el impacto de la tecnología en la vida de los niños y jóvenes de hoy está contribuyendo de forma significativa al proceso de conformación de su identidad como individuos y como generación, pues han nacido y crecido en la cibercultura.

Abordar la delicada complejidad de la constitución de la identidad en el individuo, las condiciones que la favorecen y aquellas que la limitan es una temática que concentró el esfuerzo de un grupo importante de psicólogos evolutivos, expertos en cognición y sociólogos de la educación en los años 60 del siglo pasado. Apoyándose en las teorizaciones de Eric Erikson ${ }^{4}$, autores como J.E. Marcia

3 Por supuesto, la forma en la que los profesores son "formados" merece una reflexión completamente distinta al propósito de este estudio, por lo que, para evitar una dirección necesaria e importante sobre el impacto de la formación de profesores en su futuro desempeño profesional, en función del propósito de este artículo se planteará la influencia de "los iguales" (los nativos) como posible efecto, entendiendo claramente que esta influencia requiere de una formación explícita.

4 Autor del Desarrollo Psicosocial: enfatizó la comprensión del "yo" e investigó el impacto de la cultura, la sociedad y la historia en la personalidad. 
(1980) y A.S. Waterman (1984), entre otros, hicieron del desarrollo del adolescente su objeto de estudio. La "crisis" que, se creía, debía pasarse en la adolescencia, viene a ser en realidad solo una forma de manifestación que genera cambios en la personalidad del sujeto, hasta llevarlo de un estado de dependencia, a uno de independencia de sí mismo. La personalidad se construye a partir de la interacción social, pues sin el otro es imposible entender y construir una identidad. Los otros le dan significado y dotan al sujeto de los elementos que le permiten inscribirse en el seno de una sociedad, dotada de una serie de características que la hacen única y la diferencian de los demás grupos sociales (Alvarado \& Capetillo, 2017).

Martuccelli y Singly consideran que "el individuo no puede tener una cierta consistencia sino dependiendo de los juicios de los otros, de su validación" (2012, p. 69), es decir, de la mirada de los otros significativos (OS). Sin embargo, la relación entre el yo que busca individualizarse y los OS es intrínsecamente inestable en las sociedades contemporáneas, puesto que pende del equilibrio entre la referencia y el apoyo que brindan estos -que es central para la construcción de la propia identidad- y la necesidad de vivir esa propia identidad como algo que no depende de los demás (Martucelli \& Singly, 2012, p. 70). Así, en el marco del proceso de individualización, "los lazos personales con vocación electiva y contractual" y "el reconocimiento interpersonal" (Martucelli \& Singly, 2012, p. 72) se vuelven cuestiones centrales (Lemus, 2017) si realmente tenemos la intención de comprender la forma en la identidad, cibercultura y educación requieren entrelazarse.

Por otra parte, si Piaget afirmaba que el proceso socioafectivo y el intelectual son inseparables, en el sentido de que ambos funcionan en forma paralela e interactiva, podría seguirse entonces que a cada cambio cognoscitivo podría corresponder uno y viceversa (Schalk, 1992). Sin embargo, esto no se ha manifestado de forma tan ordenada y lineal como parecía en aquel tiempo, y, sin duda, la complejidad de la sociedad actual influye e impacta directamente en este proceso de construcción de la identidad de los hoy adolescentes, quienes nacen y crecen en la cibercultura. 


\section{Cibercultura y formas de relación en la construcción de la identidad}

La cibercultura, según Lévy (2000), es el conjunto de técnicas materiales e intelectuales, prácticas, actitudes, modos de pensamiento y valores, desarrollados conjuntamente en el crecimiento del ciberespacio y el oceánico universo de información y de seres humanos que navegan por él y lo alimentan.

Sobre esta base, la idea de "cibercultura" es un constructo social que mezcla la realidad "palpable" con la virtual, dejando la privacidad al descubierto en todas estas redes de comunicación interpersonal; las ideas de "cultura", "cibercultura", "tecnología", "tiempo", "espacio" están construidas socioculturalmente. Nada es "natural en el mundo social; las formas de ver y percibir tampoco" (Algueda \& Gaete, 2016).

Por otra parte, debido a la tecnología, emerge un nuevo tipo de cultura propia (digital) que se desarrolla en el ciberespacio, creado a partir de la interconexión mundial de ordenadores que impulsan la conjunción entre las prácticas online y las offline de la acción (Méndez, 2015).

Investigaciones recientes han retomado la propuesta analítica de Martuccelli, para analizar la experiencia de las juventudes contemporáneas, en orden de identificar sus relaciones afectivas y la forma como dan fundamento a su vida (Lemus, 2017). Por su parte, Abril \& León (2016), sobre la base de lo aportado por Urresti (2008), señalan que este tipo de relaciones a través del ciberespacio van forjando su autoconcepto y del lugar al que pertenecen. Reguillo (2012) considera que las tecnologías intervienen en la constitución de sus identidades y moldean y significan sus prácticas cotidianas, así como en las maneras en que se vinculan e interactúan con el mundo, tales como las formas de socializar y consumir su cultura, y vivir procesos de aprendizaje y experiencias de organización política (Lemus, 2017). 
La pantalla se ha vuelto un accesorio no solamente desde el punto de vista tecnológico, sino también desde los procesos culturales, pues la comunicación e interacción se hace a través de redes sociales, como Twitter, Facebook, YouTube o Skype. Más que un accesorio iconográfico, la pantalla se vuelve una herramienta que contribuye a la construcción de la identidad de los jóvenes en la red (Torres, 2016).

Sin embargo, así como las tecnologías no "enseñan” en sí mismas, tampoco realizan los cambios en las personas ni en las sociedades en las que aquellas viven. Por ello su utilización va más allá de su uso eficiente, y está inserta en una cultura y en un contexto histórico específico que fundamentan el propósito de su uso: "el proyecto se interpone al artefacto, sea este un periódico gráfico, una radio, una producción audiovisual, un sitio web o las redes sociales" (Méndez, 2015, p. 3).

Es así como se hace necesario y significativamente relevante que la educación anticipe el conjunto de estrategias y acciones que permita dotar a los jóvenes de un pensamiento crítico robusto, disciplinado y bien fundamentado, para vivir el complejo proceso de constitución de su identidad en entornos altamente "tecnologizados" en los que se ven expuestos y envueltos, en los que imperan la apropiación social de internet, el activismo en la calle y el activismo en el espacio virtual; la estética y culturización de la práctica política, la diáspora de posiciones políticas e ideológicas (Méndez, 2015), así como las posverdades, las contraverdades, las deliberadas mentiras, los relativismos y las incongruencias, la confusión entre temas emergentes y valores, los desdibujos en el balance de las libertades y las responsabilidades. Todo esto les ofrece "la imagen" de ser protagonistas, cuando pueden llegar a ser solo parte de la cadena de reproducción de aquello que denuncian, sin siquiera darse cuenta de ello, y configurando una imagen de sí mismos tan distante como frágil.

Esta sobreexposición no solo debilita, sino que además infiere un grado de confusión importante entre el proceso de la individualiza- 
ción y el que se produce como resultado de la natural presión que ejerce "pertenecer" y "ser visto y reconocido" en la cibercultura.

Las redes sociales son transversales, multirramificadas y permiten construir distintas identidades y distintos " $y o$ " para satisfacer los deseos que parecen inalcanzables en la cotidianidad, construyendo un perfil que se amplía en la realidad virtual y que se vuelve paralelo a la realidad física (Torres, 2016). Entonces los jóvenes comienzan a moverse en dos mundos distintos respecto de sí mismos: quiénes son realmente y quiénes ellos anhelan ser para pertenecer (Guerrero, 2015).

Moreno (Lemus, 2017) asegura que el motor de adherencia a Facebook es la vida social que allí se desarrolla, la moda y la presión ejercida desde el grupo de pares, el deseo de no quedarse afuera. Solo como un ejemplo de ello podemos advertir algunos hallazgos que se observan en la vida de los jóvenes, que reinventan el espacio público como expresión de identidad: del "yo soy", "yo vivo", y de la representación de su propia vida. Una representación que no siempre es reflejo de su realidad y que confunde la construcción interna de su identidad igualándola a la imagen que crea de sí mismo para poder o querer pertenecer (Alvarado \& Capetillo, 2017).

Esta configuración de espacios provoca la sensación de libertad y autonomía construida a partir de la interacción con sus amigos (Torres, 2016) y, a la vez, una sensación de dependencia respecto de la búsqueda de legitimación, reconocimiento y aprobación por parte de los pares, y que se vinculan con formas de actuar y presentarse en las prácticas juveniles off line (Ramírez, Galeano \& Osorio, 2014).

Es así como la cibercultura nos insta a configurar espacios educativos que ofrezcan a los jóvenes la posibilidad de mirarse de cerca y con distancia, con emocionalidad, y a su vez con un pensamiento preciso y riguroso, como individuos y como parte de una sociedad que siempre es posible mejorar, pero que no es necesario destruirlo todo para poder lograrlo. Ello requiere tolerancia, capacidad de diálogo, respeto y compromiso inclaudicable consigo 
mismos y con los otros, como "legítimos otros" (Maturana, 2002), para que esta relación del "sí mismo" con "los demás" (todos los demás) sea el cimiento constituido sobre bases sólidas, de la sociedad que sueñan.

$\mathrm{Y}$ es aquí donde los educadores migrantes digitales tenemos no solo la oportunidad sino la responsabilidad de desempeñar el papel que la sociedad en su conjunto nos ha confiado, y que nosotros hemos aceptado, lo cual implica necesariamente ser flexibles para sumergirnos en esta cibercultura y saber conducirnos en ella.

\section{Educación, identidad y cibercultura}

La cibercultura también desafía a los sistemas educativos, pues afecta tanto la dimensión individual como colectiva de sus integrantes. En el ámbito individual, esto ocurre cuando sus límites traspasan la intimidad de las personas, la exacerbación de la sensibilidad (o insensibilidad) ante los fenómenos sociales, los peligros ante redes internacionales de agresión, violencia y denigración de lo humano. En su dimensión colectiva, la cibercultura presenta la manipulación de los medios de comunicación, la cultura del consumismo y la expansión de ideologías en contra de la dignidad de la humanidad (Cifuentes, 2017).

Es por ello que, tanto la escuela como el sistema educativo, están llamados a ser parte activa en la construcción de la identidad de los jóvenes para que, de forma explícita, sistemática e intencionada, puedan provocar en la juventud las reflexiones y transformaciones necesarias que les permitan discernir, argumentar y actuar congruentemente frente a los distintos hechos que acontecen en su entorno.

Vygotsky concibe la teoría relacionada fundamentalmente con la interacción social, definida como "un factor de primer orden en el desarrollo", por lo que adquiere vital importancia, pues incorpora un cambio importante en las comunicaciones, al entregarle al lector el real control de lo que consume y posiciona la interacción como eje central de la misma (Ramírez, Galeano \& Osorio, 2014). 
Un elemento imprescindible en la gestión, tanto institucional como de las políticas públicas, será entonces la de promover en los centros educativos ambientes digitales éticos que fundamenten la reflexión crítica del uso de la tecnología, el acceso, la inclusión y la formación para la formación de ciudadanos. Es necesario divulgar y practicar el uso de la "netiqueta" (Cifuentes, 2017), es decir, la base fundamental de "escucha”, respeto y códigos sociales de interacción que promuevan una positiva y constructiva relación consigo mismo y con los demás. Para ello, el fortalecimiento de la lengua hablada y escrita es un desafío aún pendiente de resolver y urgente de abordar en nuestros países, ya que, si consideramos los resultados de estas habilidades básicas de la cognición, podremos constatar que, sin una base de dominio del uso del lenguaje, las posibilidades de cimentar un pensamiento crítico-reflexivo y de su consecuente manifestación en interacciones tanto individuales como colectivas sencillamente serán nulas.

Indudablemente, en una sociedad tan penetrada por las TIC es decisivo desarrollar las habilidades comunicativas, en aras de que las personas se apropien más activamente y de forma más crítica y reflexiva de estas tecnologías, así como del desarrollo de la autonomía, la autodeterminación, la potenciación de destrezas cognitivas, entre otras (Álvarez \& Vélez, 2015).

Por otra parte, la formación de una ciudadanía (más allá de entenderla como el ejercicio de manifestación de una específica corriente política), es un reto en relación con la competencia comunicativa intercultural. Se piensa en la formación de un ciudadano, entendido como un ser humano que entra en relación con otros, llamado a construir su propia identidad y abierto a poner en diálogo sus procesos de identificación y a reconocer respetuosamente a otros. En este sentido, la escuela tiene todo por hacer en estas nuevas dinámicas de la vida social global, que integran política, sociedad, religión, estética, ciencia, cultura y ética, y que son mediadas por la tecnología (Ramírez, Galeano \& Osorio, 2014). 
Por tanto, entre los desafíos para abordar están: el fortalecimiento de la capacidad comunicativa, tanto en lenguaje hablado como escrito, el fortalecimiento de las habilidad cognitivas sobre las que el pensamiento crítico se fundamenta; el fortalecimiento de una cultura escolar o institucional basada en la ética; la creación de un ambiente educativo que entienda diversidad y su necesaria aceptación de todos, efectivamente de todos, y una formación ciudadana plural, veraz y democrática en su sentido más limpio y puro.

Una experiencia que está dando resultados muy positivos en la formación de jóvenes, en distintos países, es RULER, el programa de Inteligencia Emocional de la Universidad de Yale, el que ha logrado constatar, con evidencia fundamentada, los buenos resultados de esta iniciativa.

El desafío ahora es volver a reflexionar acerca de aquellos conceptos que daban sentido a las prácticas, y que ahora resultan imprecisos para dar cuenta de esta nueva realidad (Chávez, González \& Lay, 2017) y en el que la participación de los jóvenes puede llegar a ser fundamental.

Es decir, abordar los desafíos de todo lo anteriormente planteado, haciendo parte protagonista a los jóvenes en la construcción de la apropiación y su conducción, permite cuando menos: favorecer, en función de su propio proceso formativo, la posibilidad de organizarse en grupos para el intercambio de materiales propios de la cultura mediática, generar junto con ellos procesos, e instaurar espacios virtuales de sociabilidad aprovechando su facilidad para adaptarse a los rápidos avances tecnológicos y adquiriendo habilidades y competencias en el proceso (Abril \& León, 2016).

Por otra parte, es necesario que los sistemas de educación consideren los cambios que se experimentan con cada vez mayor fuerza respecto de la relación con el conocimiento. Por una parte, la dinámica vigencia del conocimiento; por otro, la naturaleza del trabajo con una cada vez mayor importancia en la transacción de conocimientos (sociedades del aprendizaje) y una presencia cada vez mayor de instrumentos tecnológicos que están modificando 
nuestras funciones cognitivas, tales como la modelización de fenómenos complejos, los hiperdocumentos y la inteligencia artificial. Estos fenómenos que quizás prontamente impacten el proceso de construcción de la identidad de los jóvenes.

Ciertamente se ven lejanos aún del entorno educativo, pero el llamado es a no repetir la experiencia de hace apenas veinte años, cuando señalábamos con certeza absoluta que las tecnologías serían "una moda" que pasaría prontamente. Nunca imaginamos en aquel tiempo lo distantes que estábamos de comprender la realidad. Aprendamos de esa experiencia y preparemos nuestros sistemas educacionales, nuestras políticas públicas, nuestras escuelas y, de forma central, a nuestros profesores y estudiantes para ello. Pero, por sobre todo, tomemos el desafío de preparar a nuestros jóvenes (futuros tomadores de decisiones de nuestras sociedades) para ofrecerles una educación que sea un faro que les ayude a navegar en las, a veces torrentosas, aguas de la cibercultura, y que ello los conduzca en la construcción interna de su identidad.

Esta construcción puede lograrse con una base firme de pensamiento crítico, con el lenguaje como fundamento; pero también es posible potenciar el pensamiento creativo. ¿Cómo? Abriendo la toma de decisiones pedagógicas e incorporando a los jóvenes en la forma como necesitamos enseñarles, pero considerando también cómo ellos quieren y necesitan aprender. Para ello, sus condiciones "innatas" de uso de la tecnología y de experiencia previa en la cibercultura puede, realmente ser un aporte para los profesores migrantes digitales, logrando combinar el talento, el conocimiento y la experiencia de éstos, con la innovación, creatividad y dominio tecnológico de aquellos, logrando dinamizar lo que aún nos falta por lograr: transformar las salas de clases en espacios de construcción social e individual de conocimiento, de una forma cada vez más cercana y acorde a la realidad que estos jóvenes enfrentarán en su experiencia de inserción al mundo del trabajo. 


\section{Conclusión}

La construcción de la identidad de los adolescentes y jóvenes ha sido un tema de interés en la investigación científica de las sociedades occidentales desde los años 60 del siglo anterior. Sin embargo, este conocimiento ha sido significativamente impactado por la tecnología y la instalación de la cibercultura.

Los jóvenes nativos digitales han logrado desarrollar un nivel de dominio del uso de la tecnología que supera de forma significativa a la de aquellos adultos que los conducen y los educan. A su vez, se ven expuestos a un conjunto de fenómenos en la cibercultura que requieren de la orientación de aquellos.

Entre los desafíos y oportunidades que tienen los educadores se encuentran el fortalecimiento de la capacidad comunicativa, tanto en lenguaje hablado como escrito; el fortalecimiento de las habilidad cognitivas sobre las que el pensamiento crítico se fundamenta; el fortalecimiento de una cultura escolar o institucional basada en la ética; la creación de un ambiente educativo que entienda la diversidad y su necesaria aceptación de todos, y efectivamente de todos, una formación ciudadana plural, veraz y democrática en su sentido más limpio y puro.

\section{Bibliografía}

Abril, J. \& León, G. (2016). Nueva cultura audiovisual y Ciberculturas juveniles: sociabilidad y apropiación mediática de jóvenes en el ciberespacio. Revista Internacional de Cultura Visual, $3(2), 1-9$.

Algueda, F. \& Gaete, V. (2016). Fetichismo Tecnológico en la Era de la Cibercultura. Revista Educación y Tecnología, 2(09), 1625.

Alvarado, M. \& Capetillo, C. (2017). La formación de la identidad a través de las redes sociales: análisis mediante la etnografía virtual. Repositorio Institucional Caxán, 1-6.

Álvarez, G. \& Vélez, C. (2015). Configuración de subjetividades en los jóvenes universitarios sobre las Tecnologías de la Información, la Comunicación y del Aprendizaje (TIC/ TAC). Itinerario Educativo, 29(65), 223-263. 
Cifuentes, J.-E. (2017). Axiología de la Cibercultura: posibilidades y límites de la escuela. Revista de la Universidad de La Salle, 73-81.

Chávez, B., González, R. \& Lay, I. (2017). Desafíos de la cultura digital para la educación. Guadalajara, Jalisco, México: UDG Ediciones.

Garay, L. (2017). Jóvenes universitarios, participación en redes sociales digitales y temas ciudadanos, en B. Chávez, R. González \& I. Lay, Desafíos de la cultura digital para la educación (pp. 41-60). Guadalajara, Jalisco, México: Universidad de Guadalajara.

Georges, C. \& Veytia, M.-G. (2017). Nativos e Inmigrantes digitales riesgo de la conceptualización simplista, en B. Chávez, R. González \& I. Lay, Desafíos de la cultura digital para la educación (pp. 235-256). Guadalajara, Jalisco, México: Universidad de Guadalajara.

Guerrero, D. (2015). Procesos de subjetivación de jóvenes en la red social Facebook. Tesis, Universidad Distrital Francisco José de Caldas, Facultad de Posgrado, Bogotá.

Lemus, M. (2017). Jóvenes frente al mundo: Las tecnologías digitales como soporte de la vida cotidiana. Revista Latinoamericana de Ciencias Sociales, Niñez y Juventud, 15(1), 161-172.

Lévy, P. (2000). La Cibercultura y la educación. Pedagogía y Saberes, (14), 23-32.

Manzano, B. (2016). Cibercultura, TIC, Redes Sociales: nuevas formas de comunicación para las familias. Pixel Bit, 49, 195-206.

Marcia, J.-E. (1980). Identity in Adolescence. Handbook of Adolescent Psychology.

Martucelli, D. \& Singly, F. (2012). Les sociologies de l'individu: Domaines et approches. Paris, France: Armand Colin.

Maturana, H. (2002). El sentido de lo humano. Santiago: Dolmen.

Méndez, A. (2015). Nuevas culturas y estéticas en la Sociedad Red: Cibercultura contra el especismo. VIII Jornadas de Jóvenes Investigadores del Instituto de Investigaciones Gino Germani (pp. 1-15). Buenos Aires: Instituto de Investigaciones Gino Germani.

Prensky, M. (2001). Digital natives, digital inmigrants: do they really think differently. On the Horizon, 9(6).

Ramírez, M., Galeano, C. \& Osorio, L. (2014). Competencias Ciudadanas en la interacción de jóvenes en las redes sociales: Facebook y Twitter. Tesis Magíster, Universidad Pontificia Bolivariana, Facultad de Educación, Bogotá. 
Schalk, A. (1992). Identidad, Adolescencia y Pensamiento Crítico. Sinéctica, 1(1), 1-7.

Torres, C. (2016). La representación virtual del yo en los jóvenes. Revista Realidad, 47, 153-167. 